\title{
Expression and mechanism of microRNA 195 in diabetic retinopathy
}

\author{
Li Shan ${ }^{1)}$, Haiyong Zhang ${ }^{2) *}$, Ying Han $^{3)}$ and Ruijuan $\mathrm{Kuang}^{4)}$ \\ 1) Department of Endocrinology, Zibo Central Hospital, Zibo, Shandong, China \\ 2) Department of Pulmonary Disease, Binzhou Hospital of Traditional Chinese Medicine, Binzhou, Shandong, China \\ 3) Department of Endocrinology, Zouping People's Hospital, Zouping, Shandong, China \\ 4) Department of Endocrinology, Jiaozhou People's Hospital, Jiaozhou, Shandong, China
}

\begin{abstract}
We aimed to explore the role of microRNA 195 (miR-195) in diabetic retinopathy (DR). From January 2019 to July 2020, 50 patients with DR undergoing vitrectomy and 40 patients with idiopathic macular holes undergoing vitrectomy were selected as the observation group (OG) and control group (CG), respectively. The mRNA and protein expression levels of miR-195, SIRT1, BAX, and BCL-2 were detected in the retinal tissues obtained from the two groups during surgery. In addition, human retinal endothelial cells and human dermal microvascular endothelial cells were cultured in a high-glucose environment to detect the targeted relationship between miR-195 and SIRT1; determine the mRNA and protein expression levels of SIRT1, BAX, and BCL-2 after miR-195 knockdown; and assess the levels of cell proliferation and apoptosis. In OG, the mRNA and protein expression levels of miR-195 and BAX were high, whereas those of BCL-2 and SIRT1 were low. Moreover, we detected a targeted relationship between miR-195 and SIRT1. Conversely, miR-195 knockdown led to the downregulation of the mRNA and protein expression levels of BAX and the upregulation of the mRNA and protein expression levels of SIRT1 and BCL-2 as well as improvement in cell growth and a decrease in the apoptosis rate. miR-195 is overexpressed in DR, and its targeted relationship with SIRT1 inhibits the growth of cells in the retina and accelerates apoptosis.
\end{abstract}

Key words: microRNA 195 (miR-195), SIRT1, BCL-2, BAX, Diabetic retinopathy

DIABETES is a common disease involving hyperglycemia caused by defective insulin secretion function $[1,2]$. It is estimated that 600 million people will suffer from diabetes by 2040 , and more than one-third of them will suffer from diabetic retinopathy (DR) and other complications [3-5]. DR is a serious public health concern and the main cause of blindness among working-age adults worldwide. The loss of vision caused by this disease affects the quality of life of patients and negatively impacts the psychology of the patients and society [6]. The symptoms of DR include hemorrhage, microaneurysms, cotton wool spots, lipid exudates, macular edema, capillary occlusion, and neovascularization, all of which can eventually lead to blindness [7, 8]. Therefore, it is important to elucidate the pathogenesis, basic mechanism, and treatment of DR.

Submitted Apr. 28, 2021; Accepted Nov. 10, 2021 as EJ21-0231

Released online in J-STAGE as advance publication Dec. 23, 2021

Correspondence to: Ruijuan Kuang, No. 180, Huzhou Road, Jiaozhou, Shandong 266300, China.

E-mail: kuangruijuan@aliyun.com

*Both authors contributed equally to this work and should be considered as equal first coauthors.
Recently, SIRT1, a silent information regulator protein, has become a key molecular target for diabetes. This NAD+-dependent deacetylase plays an important role in metabolic regulation and adaptation and is widely involved in the regulation of inflammation, oxidative stress, autophagy, and apoptosis $[9,10]$. A study found that SIRT1 plays an important role in DR and is an associated molecular target [11]. SIRT1 can downregulate the ratio of proapoptotic BAX to antiapoptotic BCL-2, thus reducing apoptosis $[12,13]$. In recent years, SIRT1 inhibition by associated microRNAs (miRs) has become an important research avenue [14]. miRs can regulate mRNA by combining with the 3'-UTR of the target gene mRNA, leading to regulation of gene expression. Abnormal expression levels of miRs can easily lead to diseases $[15,16]$. miR-195 belongs to the miR-15/107 family and plays an important role in various types of cancers [17]. Moreover, it serves as a tumor suppressor that inhibits cancer cell proliferation [18]. In a study on endothelial injury, miR-195 was found to have a targeted relationship with SIRT1 [19]. However, few studies have addressed their role in DR; thus, we aimed to explore their role in DR. 


\section{Material and Methods}

\section{General information}

From January 2019 to July 2020, 50 patients with DR undergoing vitrectomy were selected as the observation group (OG) and 40 patients with idiopathic macular holes undergoing vitrectomy were selected as the control group (CG). This study was approved by the Ethics Committee of Jiaozhou People's Hospital, and informed consent forms were obtained from the participants.

The inclusion criteria for $\mathrm{OG}$ were as follows: the patient's pathological changes were caused by type 2 diabetes; repeated vitreous hemorrhage and drug treatments were ineffective; B-ultrasound revealed an epiretinal membrane and tractional retinal detachment; no obvious vitreous hemorrhage but B-ultrasound, ophthalmoscopy, or optical coherence tomography showed a hyperplastic membrane in the fundus and tractional retinal detachment. The exclusion criteria for OG were as follows: fasting blood glucose level of $>8 \mathrm{mmol} / \mathrm{L}$ or severe hypertension; a history of vitreoretinal or other eye surgery; having received antiangiogenic drugs; having retinal hyperplasia or retinal vascular disease caused by other factors; or having malignant tumor complications, immune system diseases, or acute or chronic infection.

The inclusion criterion for CG was as follows: patients without diabetes mellitus. The exclusion criteria for $\mathrm{CG}$ were as follows: patients with other eye diseases, malignant tumors, immune system diseases, or acute or chronic infections.

\section{Methods}

\section{Main reagents and experimental equipment \\ Materials}

Human retinal endothelial cells (HRECs) and human dermal microvascular endothelial cells (HMECs) were purchased from Angio-Proteomie. Dulbecco's modified Eagle's medium (DMEM) and 10\% fetal bovine serum were purchased from Hunan Fenghui Biotechnology Co., Ltd. The cell-cycle test kit was purchased from Sigma
Aldrich (Shanghai). The transfection reagent for the apoptosis test kit (Lipofectamine TM3000) was purchased from Thermo Fisher (China). TRIzol reagent was purchased from Yuanye Biotechnology Co., Ltd., Shanghai, China. The primer sequences and transfection plasmids for miR-195, SIRT1, BAX, BCL-2, and internal reference were designed at Sangon Biotech, Shanghai (Table 1).

\section{Instruments}

The UV spectrophotometer was purchased from Beijing Jiayuan Industrial Technology Co., Ltd. The CoulterCytoFLEX instrument was purchased from Beckman Coulter, USA. The ABI7500 fluorescence quantitative polymerase chain reaction (qPCR) instrument was purchased from Beijing Long Jump Biological Science and Technology Development Co., Ltd. The transwell chamber was purchased from Shanghai Shengbo Biomedical Technology Co., Ltd, and the microplate reader was purchased from Beijing Image Trading Co., Ltd.

\section{Detection of $m R N A$ expression levels of miR-195, SIRT1, $B A X$, and BCL-2}

The mRNA expression levels of miR-195, SIRT1, BAX, and BCL-2 were determined in retinal tissues (proliferative membrane) collected from $\mathrm{OG}$ and $\mathrm{CG}$ during surgery using qPCR. For this, total RNA in the cells was extracted by placing $\sim 50 \mathrm{mg}$ of tissues in a 1.5-mL RNase-free centrifuge tube, followed by adding $0.5 \mathrm{~mL}$ of TRIzol (Invitrogen, Burlington, ON, Canada), shaking, adding another $0.5 \mathrm{~mL}$ of TRIzol, and incubation; the entire process was completed in approximately 30 min. Subsequently, $200 \mu \mathrm{L}$ of chloroform was added to every $1 \mathrm{~mL}$ of TRIzol, shaken quickly and mixed evenly for $30 \mathrm{~s}$, and then placed on ice for $5 \mathrm{~min}$. The tube was then centrifuged at $1,500 \mathrm{~g}$ at $4^{\circ} \mathrm{C}$ for $10 \mathrm{~min}$. Using a pipette, $\sim 400-600 \mu \mathrm{L}$ of the supernatant was transferred to a new centrifuge tube and $500 \mu \mathrm{L} / 1 \mathrm{~mL}$ of TRIzol isopropanol was added. The tube was then capped, mixed by repeated inversion, incubated for $10 \mathrm{~min}$, and centrifuged at $1,500 \mathrm{~g}$ at $4^{\circ} \mathrm{C}$ for $10 \mathrm{~min}$. The supernatant was discarded, isopropyl alcohol was absorbed, and

Table 1 Primers used in this study

\begin{tabular}{lcc}
\hline Factor & \multicolumn{1}{c}{ Upstream Primers } & Downstream Primers \\
\hline miR-195 & 5'-GGCTAGCAGCACAGAAAT-3' & 5'-GTGCAGGGTCCGAGGT-3' \\
SIRT1 & 5'-GAGAGACGTCTGGTAGATCG-3' & 5'-GTGCCAGCATGTGTCGTAGT-3' \\
BAX & 5'-GCCCTTTTGCTTCAGGGTTT-3' & 5'-TCCAATGTCCAGCCTTTG-3' \\
BCL-2 & 5'-CGGAGGCTGGGATGCCTTTG-3' & 5'-TTTGGGGCAGGCATGTTGAC-3' \\
$\beta$-actin & 5'-CCGTTCCGAAAGTTGCCTTTT-3' & 5'-ATCATCCATGGTGAGCTGGC-3' \\
\hline
\end{tabular}


$1 \mathrm{~mL}$ of $75 \%$ ethanol was added and mixed well. These samples were centrifuged at $1,500 \mathrm{~g}$ at $4{ }^{\circ} \mathrm{C}$ for $10 \mathrm{~min}$, and the RNA was washed. The supernatant was discarded, the pellet was air dried for 5-10 min, and $20 \mu \mathrm{L}$ of diethylpyrocarbonate-treated water was added to completely dissolve the total RNA. Real-time (RT)-PCR was performed using a High Capacity cDNA Reverse Transcription kit (Applied Biosystems, Foster City, CA, USA) and a Qiagen One Step RT-PCR kit with custommade SIRT1 and miR-195 primers and LightCycler (Roche Diagnostics, Laval, QC, Canada), as described. qPCR was performed using ABI7500 fluorescence qPCR instrument in the following steps: predenaturation at $95^{\circ} \mathrm{C}$ for $5 \mathrm{~min}$, denaturation at $95^{\circ} \mathrm{C}$ for $15 \mathrm{~s}$, and annealing at $60^{\circ} \mathrm{C}$ for $30 \mathrm{~s}$ and $60^{\circ} \mathrm{C}-95^{\circ} \mathrm{C}$ for a total of 40 cycles. Finally, miR-195 and SIRT1 obtained were compared with the internal reference.

\section{Detection of the protein expression levels of SIRT1, $B A X$, and $B C L-2$}

For each sample, $25 \mu \mathrm{g}$ of total protein extracts were separated on SDS-PAGE gels and transferred to polyvinylidene difluoride membrane. The expression ratio levels of SIRT1 (Abcam, ab189494, 1:1,000), BAX (Abcam, ab32503, 1:1,000), and BCL-2 (Abcam, ab32124, 1:1,000) to the reference materials GAPDH (Abcam, ab9485, 1:1,000) was considered the relative expression level.

\section{Cell culture and transfection}

In a cell incubator, HRECs and HMECs were routinely subcultured in high-glucose DMEM medium containing $10 \%$ fetal bovine serum at $37^{\circ} \mathrm{C}$ with $5 \% \mathrm{CO}_{2}$. Before transfection, the cells were seeded in 96-well plates and then divided into miR-NC and miR-195 mimic groups. Transfection was conducted using Lipofectamine TM3000, and the mRNA and protein expression levels of miR-195, SIRT1, BAX, and BCL-2 in each group of cells were tested according to previous methods.

\section{Detection of cell proliferation}

The two groups of transfected HRECs and HMECs were each inoculated into 96-well plates, and each well was provided with three multiple holes, which were set at three time points, i.e., 24, 48, and $72 \mathrm{~h}$. Then, $2 \mathrm{~h}$ before the end of the culture period, $20 \mu \mathrm{L}$ of MTS Cell Proliferation Colorimetric Assay Kit (CCK8) reagent was added to each well and the plates incubated at $37^{\circ} \mathrm{C}$ with $5 \% \mathrm{CO}_{2}$. After $2 \mathrm{~h}$, the optical density was measured at a wavelength of $490 \mathrm{~nm}$ using an automatic microplate spectrophotometer to observe cell proliferation.

\section{Detection of apoptosis}

A CoulterCytoFLEX flow cytometer was used to detect cells that were transfected for $48 \mathrm{~h}$ in a 6-well plate and stained with annexin V and PI. This experiment was repeated three times. The cells were then analyzed using the Fluorescent-Activated Cell Sorter (BD FACSaria cell sorter, BD Bioscences).

\section{Detection of luciferase activity}

A dual-luciferase reporter gene was used to detect the targeted relationship between miR-195 and SIRT1. HRECs and HMECs were seeded in a 96-well plate and cells in the miR-195 inhibitor group, SIRT1-Mut and SIRT1-Wt were transfected with Lipofectamine TM3000. All constructs were verified by sequencing. The underlined nucleotides (MI0000489) indicated the bases where mutations were made. According to the manufacturer's instructions, the luciferase activities of fireflies and sea kidneys were analyzed using the DualLuciferase Reporter Assay (Promega). For luciferase assay, 293A cells were cultured in 24-well plates and transfected with $100 \mathrm{ng}$ luciferase reporter plasmid, $5 \mathrm{ng}$ pRL-TK vector expressing the Renilla luciferase (Promega), and $50 \mathrm{pmol} / \mathrm{L}$ of miR-195 inhibitor group, SIRT1-Mut, SIRT1-Wt, or negative control precursor. After transfection for $36 \mathrm{~h}$, firefly and renilla luciferase activities were measured using the Dual-Luciferase Reporter Assay (Promega). Each transfection was repeated twice in triplicate.

\section{Statistical analysis}

We used SPSS 19.0 (Asia Analytics, formerly SPSS China) for statistical analysis. The measurement data were expressed as (Mean \pm S.D), and $t$-test and one-way analysis of variance were used for analysis. The least significant difference method was used for post-hoc analysis. Significance was set at $p<0.05$.

\section{Results}

\section{Clinical characteristics}

No difference in age was found between OG (61.5 \pm 9.8 years $)$ and $\mathrm{SG}(60.2 \pm 10.1$ years $)$; half of the patients were women in each group. Body mass index was not different between OG $(29 \pm 5)$ and SG (30 \pm 4 ). The duration of diabetes mellitus was $19 \pm 6$ years in $\mathrm{OG}$ and the duration of diopathic macular holes was $13 \pm 5$ months.

\section{Correlation of miR-195 with SIRT1 in the retinal tissues of patients}

A comparison of the expression levels of miR-195 and SIRT1 between the groups revealed that miR-195 
expression level in OG was higher than that in $\mathrm{CG}$, whereas the SIRT1 mRNA and protein expression levels were further decreased in $\mathrm{OG}(p<0.05)$ (Fig. 1).

\section{$B A X$ and $B C L-2$ in retinal tissues}

OG exhibited increased levels of BAX and BCL-2 compared with $\mathrm{CG}(p<0.05)$ (Fig. 2).

\section{Targeted relationship between miR-195 and SIRT1}

According to the related literature [20], we predicted the binding site between miR-195 and SIRT1, and the results of Dual-Luciferase Reporter Assay of HRECs and HMECs showed that the luciferase activity of SIRT1-Wt of miR-195 inhibitors increased, whereas that of SIRT1Mut did not vary $(p<0.05)$ (Fig. 3).

\section{Effect of miR-195 knockdown on SIRT1 expression level}

miR-195 was knocked down in the two cell lines, and it was found that the expression levels of miR-195 and
A

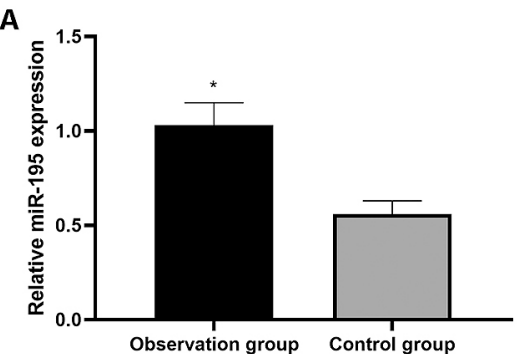

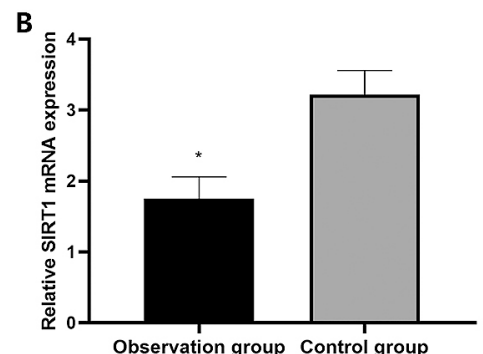

Observation group Control group

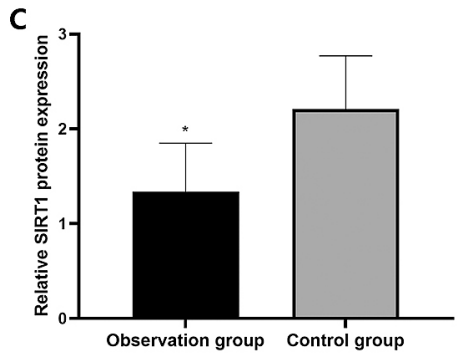

Fig. 1 Expression levels of microRNA 195 (miR-195) and SIRT1 in the two groups

(A) The miR-195 expression in the observation group (OG) was higher than that in the control group (CG) $(p<0.05)$; (B) the SIRT1 mRNA expression level in OG was lower than that in CG $(p<0.05)$; (C) the SIRT1 protein expression level in OG was lower than that in $\mathrm{CG}(p<0.05)$.

* compared with $\mathrm{CG} ; p<0.05$

A

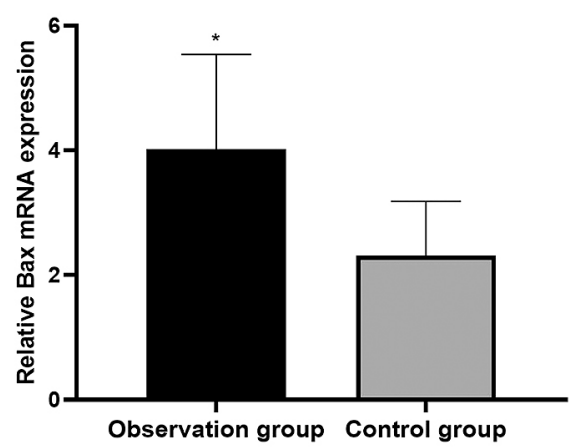

C

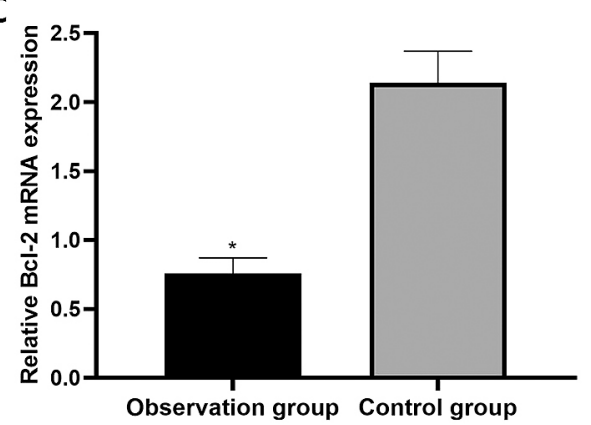

B

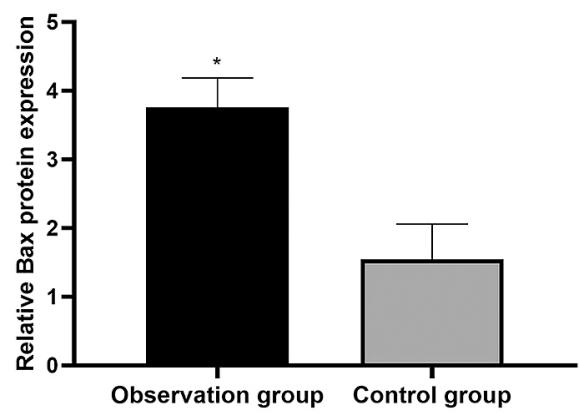

D

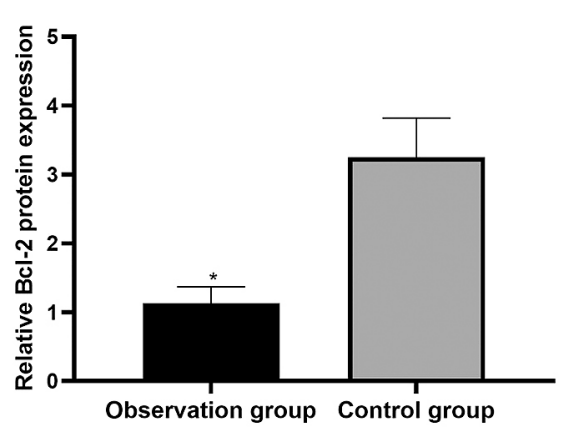

Fig. 2 mRNA and protein expression levels of BAX and BCL-21 in the two groups

(A) The BAX mRNA expression level in the observation group (OG) was higher than that in the control group $(\mathrm{CG})(p<0.05)$; (B) the BAX protein expression level in OG was higher than that in $\mathrm{CG}(p<0.05)$; (C) the BCL-21 mRNA expression level in OG was lower than that in CG $(p<0.05)$; (D) the BCL-21 protein expression level in OG was lower than that in CG $(p<0.05)$.

* compared with $\mathrm{CG} ; p<0.05$ 
SIRT1 were negatively correlated. The miR-195 expression was lower in the miR-195 inhibitor group than in the miR-NC group, whereas the mRNA and protein expression levels of SIRT1 were higher than those in the miR-NC group $(p<0.05)$ (Fig. 4).

A

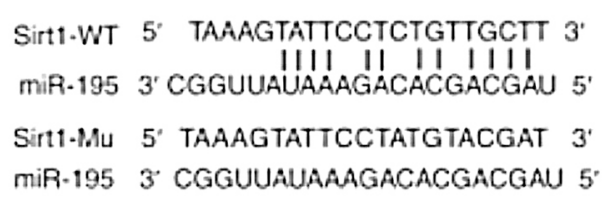

$m R N A$ and protein expression levels of $B A X$ and BCL-2 after miR-195 knockdown

A comparison of the mRNA and protein expression levels of BAX and BCL-2 revealed that the mRNA and protein expression levels of BAX in the miR-195 inhibitor group were lower than those in the miR-NC group, whereas the miR-195 inhibitor group showed increased the mRNA and protein expression levels of BCL-2
B

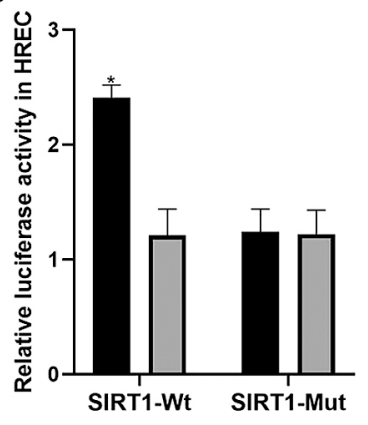

C

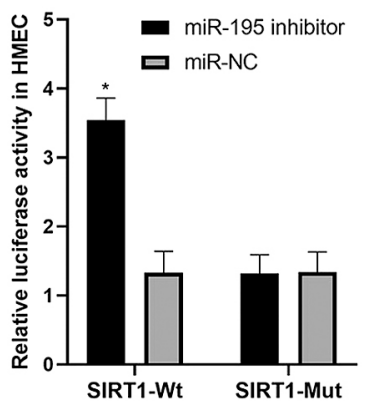

Fig. 3 microRNA 195 (miR-195) has a targeted relationship with SIRT1

(A) SIRT1 had a binding site for miR-195. (B) In HREC cells, the luciferase activity of SIRT1-Wt was higher in the miR-195 inhibitor group than in the miR-NC group $(p<0.05)$. (C) In HMECs, the luciferase activity of SIRT1-Wt was higher in the miR-195 inhibitor group than in the miR-NC group $(p<0.05)$.

\# compared with the miR-NC group; $p<0.05$

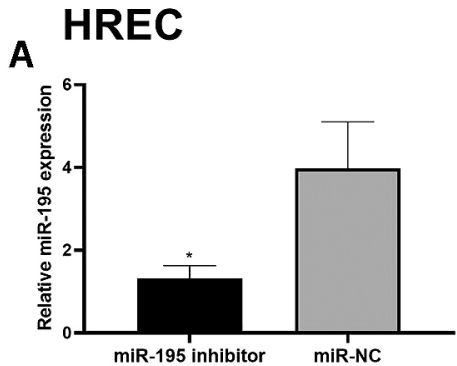

\section{HMEC}

D

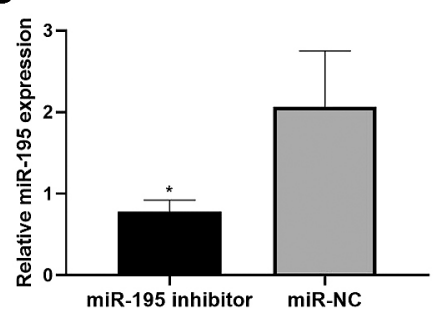

B

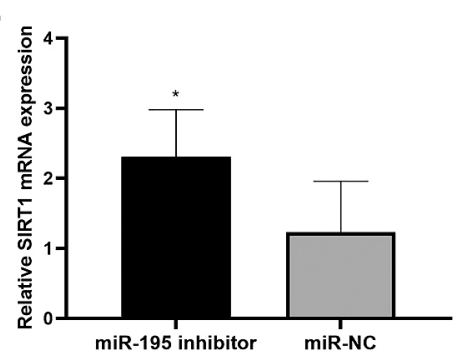

E

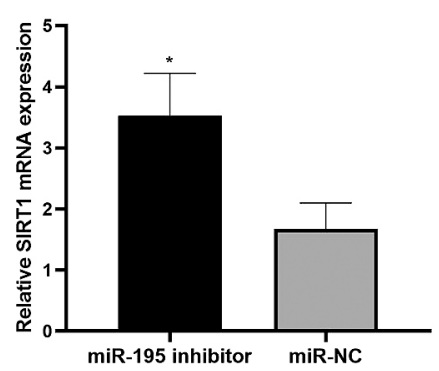

C

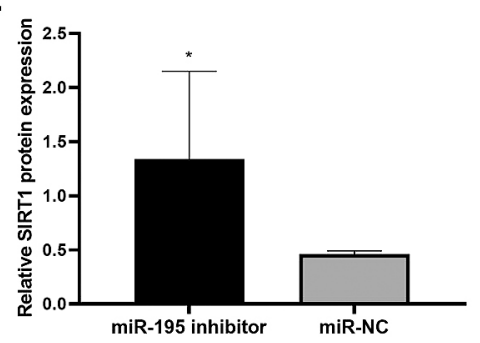

$\mathbf{F}$

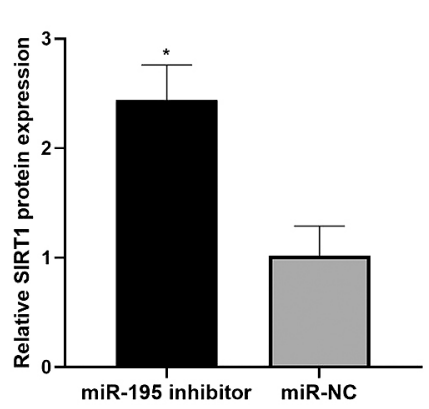

Fig. 4 mRNA and protein expression levels of microRNA 195 (miR-195) and SIRT1 in HRECs and HMECs

In HRECs, (A) the expression level of miR-195 was higher in the miR-195 inhibitor group than in the miR-NC group $(p<0.05)$;

(B) the expression level of the SIRT1 mRNA was lower in the miR-195 inhibitor group than in the miR-NC group $(p<0.05)$;

(C) the expression level of the SIRT1 protein was lower in the miR-195 inhibitor group than in the miR-NC group $(p<0.05)$.

In HMECs, (D) the expression level of miR-195 was higher in the miR-195 inhibitor group than in the miR-NC group $(p<0.05)$;

(E) the expression level of the SIRT1 mRNA was lower in the miR-195 inhibitor group than in the miR-NC group $(p<0.05)$;

(F) the expression level of the SIRT1 protein was lower in the miR-195 inhibitor group than in the miR-NC group $(p<0.05)$.

* compared with the miR-NC group; $p<0.05$. 
compared with the miR-NC group $(p<0.05)$ (Fig. 5).

\section{Effect of miR-195 knockdown on the proliferation levels of HRECs and HMECs}

A comparison of the proliferation levels of HRECs and HMECs between the two groups revealed significant differences in HREC and HMEC growth between the two groups from 24 to $72 \mathrm{~h}(p<0.05)$. The cell growth in the miR-195 inhibitor group was significantly better than that in the miR-NC group from 48 to $72 \mathrm{~h}(p<0.05)$ (Fig. 6).

\section{Effect of miR-195 knockdown on HRECs and HMECs apoptosis}

On comparing the apoptosis of HRECs and HMECs between the two groups, a decreased apoptosis rate of cells was observed in the miR-195 inhibitor group compared with the miR-NC group $(p<0.05)$ (Fig. 7).

\section{Discussion}

Diabetes is a complex metabolic disorder [21] that causes damage or dysfunction in many organs, including the heart, eyes, nerves, kidneys, and blood vessels. DR is one of the most important complications of diabetes $[22,23]$. The pathology of this disease, particularly the related score indicators, such as miRNA, has always been an important area of research [24]. In the present study, the role of miR-195 in this disease was analyzed using two approaches: patient tissue tests and cell-based experiments.

A comparison of the results obtained for patients in OG and CG revealed that the miR-195 expression were higher and those of SIRT1 were lower in OG. SIRT1 plays a role in regulating inflammation and relieving oxidative stress and vascular dysfunction in diabetes [25]. A study on palmitate-stimulated cardiomyocyte apoptosis reported that miR-195 directly targeted SIRT1 and inhibited its expression, indicating that the elevation of miR-195 inhibited the growth and proliferation of
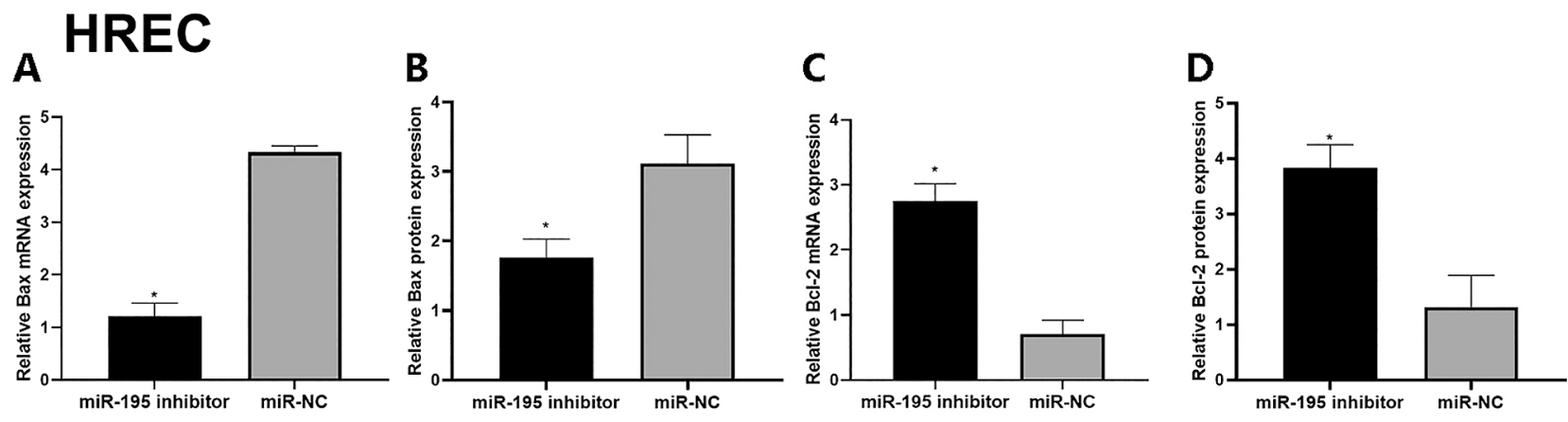

\section{E HMEC}
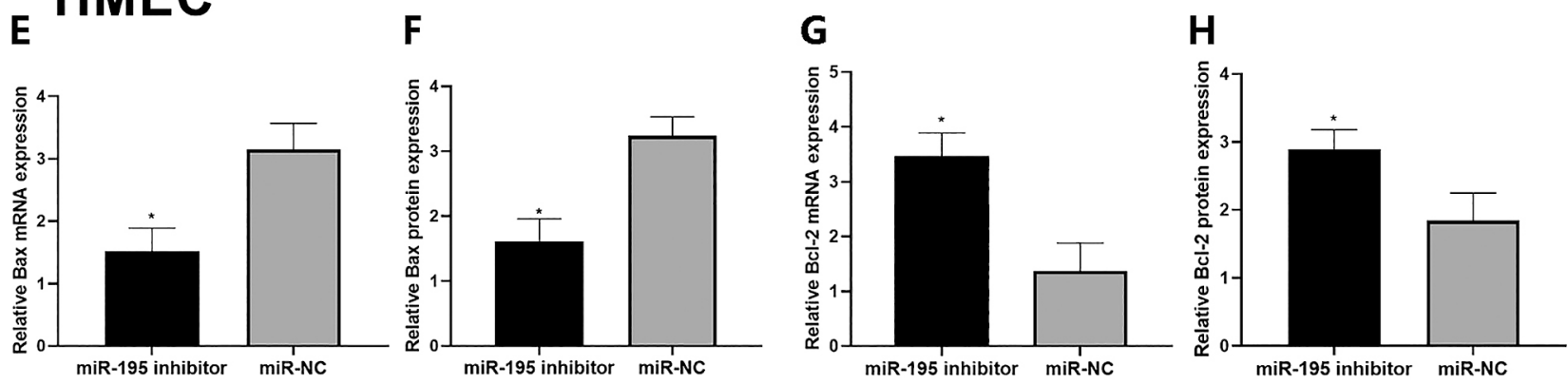

Fig. 5 mRNA and protein expression levels of BAX and BCL-21 in HRECs and HMECs

In HRECs, (A) the expression level of the BAX mRNA was lower in the microRNA 195 (miR-195) inhibitor group than in control group (CG) $(p<0.05)$; (B) the expression level of BAX protein was lower in the miR-195 inhibitor group than in CG $(p<0.05)$; (C) the expression level of the BCL-21 mRNA was higher in the miR-195 inhibitor group than in the miR-NC group $(p<0.05)$;

(D) the expression level of the BCL-21 protein was higher in the miR-195 inhibitor group than in CG $(p<0.05)$. In HMECs, (E) the expression level of BAX mRNA was lower in the miR-195 inhibitor group than in $\mathrm{CG}(p<0.05)$; (F) the expression level of BAX protein was lower in the miR-195 inhibitor group than in CG $(p<0.05)$; $(\mathrm{G})$ the expression level of BCL-21 mRNA in was higher in the miR-195 inhibitor group than in the miR-NC group $(p<0.05)$; $(\mathrm{H})$ the expression level of BCL-21 protein was higher in the miR-195 inhibitor group than in CG $(p<0.05)$.

* compared with the miR-NC group; $p<0.05$. 
A

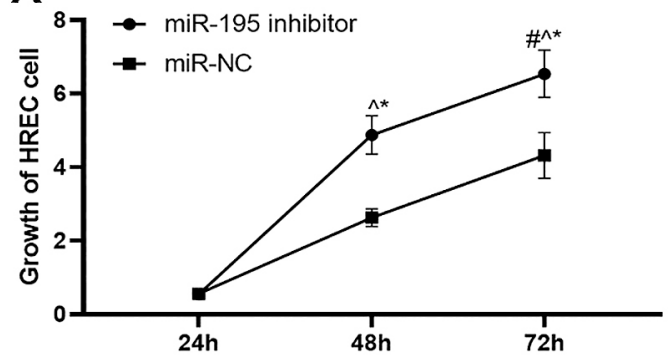

B

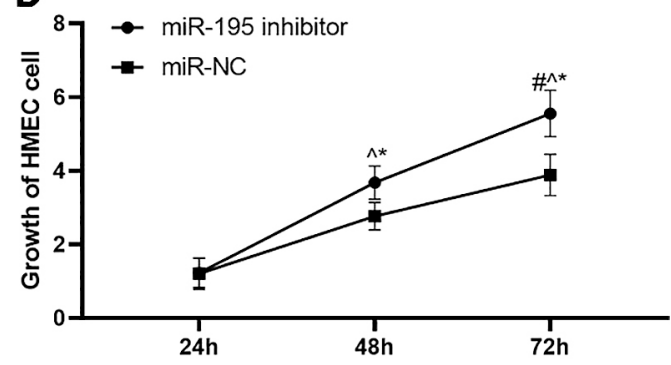

Fig. 6 Proliferative capacity of HRECs and HMECs in the two groups

(A) In HRECs, there were obvious differences in cell growth between 24 and $72 \mathrm{~h}$, and the cell proliferation was better in the microRNA 195 (miR-195) inhibitor group than in control group (CG) $(p<0.05)$; (B) in HMECs, there were significant differences in cell growth between 24 and $72 \mathrm{~h}$, and the proliferation level was better in the miR-195 inhibitor group than in CG $(p<0.05)$.

* compared with miR-NC group; $p<0.05$

* compared with $24 \mathrm{~h} ; p<0.05$

\# compared with $48 \mathrm{~h} ; p<0.05$.

A

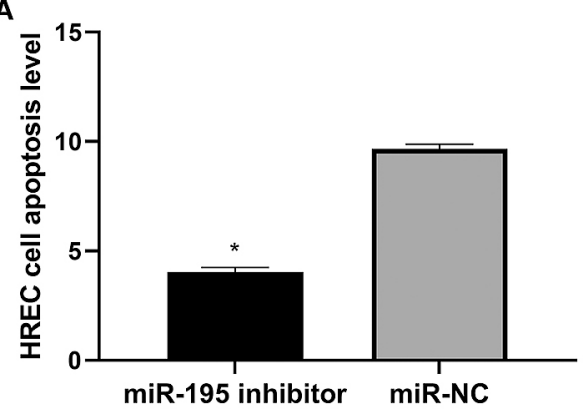

B

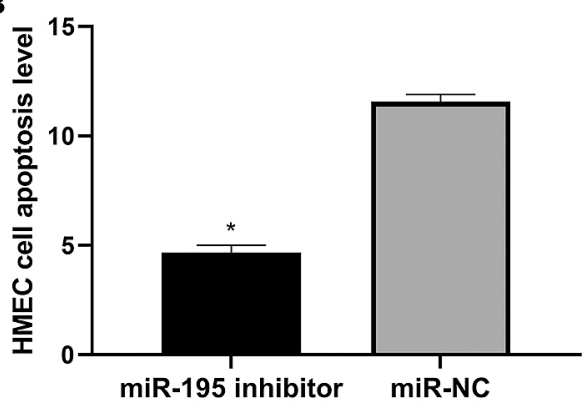

Fig. 7 Apoptosis levels in HRECs and HMECs in the two groups

(A) In HRECs, apoptosis was lower in the microRNA 195 (miR-195) inhibitor group than in control group (CG) $(p<0.05)$; (B) in HMECs, apoptosis was lower in the miR-195 inhibitor group than in CG $(p<0.05)$.

* compared with the miR-NC group; $p<0.05$

* compared with $24 \mathrm{~h} ; p<0.05$

\# compared with $48 \mathrm{~h} ; p<0.05$.

cardiomyocytes and promoted their apoptosis. This further confirms that miR-195 is an important indicator of further deterioration of the disease in the conditions of myocardial hypertrophy and heart failure [26]. In our study, the expression levels of miR-195 were higher and those of SIRT1 were lower in OG than in CG, which indicated that the increased miR-195 expression plays an important role in the aggravation of diabetes. We also suspected that SIRT1 inhibition by miR-195 is a pathogenic mechanism of diabetes, similar to its role in myocardial hypertrophy.

The results of our experiments indicated that miR-195 knockdown upregulated the expression levels of SIRT1 in HRECs and HMECs cultured in a high-glucose environment. The results also showed that BAX was upregulated and BCL-2 was downregulated in OG. The experiments performed in HRECs and HMECs revealed that miR-195 knockdown downregulated BAX, upregu- lated $\mathrm{Bcl}-2$, accelerated cell proliferation, and decreased the apoptosis rate. Antiapoptotic factors BCL-2 and BCL-xL and proapoptotic factor BAX are naturally in equilibrium in the mitochondrial pathway, which may determine the survival or death of retinal cells after diabetes stimulation [27]. Previous research found that SIRT1 inhibited BAX and increased BCL-2 [28], whereas the targeted inhibition of SIRT1 by miR-195 inhibited cell growth and promoted apoptosis [26]. A comparison of the information obtained from these articles revealed that miR-195 was upregulated in the high-glucose environment of diabetes, which inhibited SIRT1; in turn, SIRT1 has anti-inflammatory and antioxidation effects and promotes cell growth followed by a slowing down of cell proliferation and an increase in the apoptosis of retinal cells. A study on diabetic vascular diseases found that miR-195 triggered the downregulation of the protective factor SIRT1, which 
could further aggravate the endothelial damage mediated by high glucose [19]. These results are similar to those of the present study. Therefore, miR-195 can be considered a pathogenic factor for a series of complications caused by diabetes, including DR.

This study also had some limitations. Owing to time and condition restrictions, we could not compare the related factors between the two groups of patients and healthy individuals and determine their role in retinopathy unrelated to diabetes. In future research, we will address these issues as well as study the related pathological mechanisms in detail and work out a treatment plan based on miR-195.
In summary, miR-195 can be considered a pathogenic factor in DR, and its targeted inhibition of SIRT1 reduces the growth and accelerates the apoptosis of cells in the retina.

\section{Acknowledgments}

None.

\section{Disclosure}

None of the authors have any potential conflicts of interest associated with this research.

\section{References}

1. Sanyoura M, Philipson LH, Naylor R (2018) Monogenic diabetes in children and adolescents: recognition and treatment options. Curr Diab Rep 18: 58.

2. Akinci B (2019) Dapagliflozin and cardiovascular outcomes in type 2 diabetes. $N$ Engl J Med 380: 1881.

3. Ting DSW, Cheung CY, Lim G, Tan GSW, Quang ND, et al. (2017) Development and validation of a deep learning system for diabetic retinopathy and related eye diseases using retinal images from multiethnic populations with diabetes. JAMA 318: 2211-2223.

4. Dekkers CCJ, Gansevoort RT, Heerspink HJL (2018) New diabetes therapies and diabetic kidney disease progression: the role of SGLT-2 inhibitors. Curr Diab Rep 18: 27.

5. Pecoits-Filho R, Abensur H, Betônico CC, Machado AD, Parente EB, et al. (2016) Interactions between kidney disease and diabetes: dangerous liaisons. Diabetol Metab Syndr 8: 50.

6. Narayanan SP, Shosha E, Palani CD (2019) Spermine oxidase: a promising therapeutic target for neurodegeneration in diabetic retinopathy. Pharmacol Res 147: 104299.

7. Hammes HP (2018) Diabetic retinopathy: hyperglycaemia, oxidative stress and beyond. Diabetologia 61: 29-38.

8. Altmann C, Schmidt MHH (2018) The role of microglia in diabetic retinopathy: inflammation, microvasculature defects and neurodegeneration. Int J Mol Sci 19: 110.

9. Kitada M, Ogura Y, Monno I, Koya D (2019) Sirtuins and type 2 diabetes: role in inflammation, oxidative stress, and mitochondrial function. Front Endocrinol (Lausanne) 10: 187.

10. Sanz MN, Grimbert L, Moulin M, Gressette M, RuckerMartin C, et al. (2019) Inducible cardiac-specific deletion of Sirt1 in male mice reveals progressive cardiac dysfunction and sensitization of the heart to pressure overload. Int J Mol Sci 20: 5005.

11. Collin de l'Hortet A, Takeishi K, Guzman-Lepe J, Morita $\mathrm{K}$, Achreja A, et al. (2019) Generation of human fatty livers using custom-engineered induced pluripotent stem cells with modifiable SIRT1 metabolism. Cell Metab 30: 385-401.e9.
12. Nguyen PA, Won JS, Rahman MK, Bae EJ, Cho MK (2019) Modulation of Sirt1/NF-kB interaction of evogliptin is attributed to inhibition of vascular inflammatory response leading to attenuation of atherosclerotic plaque formation. Biochem Pharmacol 168: 452-464.

13. Sasaki Y, Ikeda Y, Miyauchi T, Uchikado Y, Akasaki Y, et al. (2020) Estrogen-SIRT1 axis plays a pivotal role in protecting arteries against menopause-induced senescence and atherosclerosis. J Atheroscler Thromb 27: 47-59.

14. Zeng Y, Cui Z, Liu J, Chen J, Tang S (2019) MicroRNA-29b-3p promotes human retinal microvascular endothelial cell apoptosis via blocking SIRT1 in diabetic retinopathy. Front Physiol 10: 1621.

15. Zhou HY, Wu CQ, Bi EX (2019) MiR-96-5p inhibition induces cell apoptosis in gastric adenocarcinoma. World $J$ Gastroenterol 25: 6823-6834.

16. Xiao X, Jiang Y, Liang W, Wang Y, Cao S, et al. (2019) miR-212-5p attenuates ferroptotic neuronal death after traumatic brain injury by targeting Ptgs2. Mol Brain 12: 78.

17. Lin X, Wang S, Sun M, Zhang C, Wei C, et al. (2019) miR-195-5p/NOTCH2-mediated EMT modulates IL-4 secretion in colorectal cancer to affect M2-like TAM polarization. J Hematol Oncol 12: 20.

18. Zhou S, Yu L, Xiong M, Dai G (2018) LncRNA SNHG12 promotes tumorigenesis and metastasis in osteosarcoma by upregulating Notch2 by sponging miR-195-5p. Biochem Biophys Res Commun 495: 1822-1832.

19. Qin R, Zhang L, Lin D, Xiao F, Guo L (2019) Sirt1 inhibits HG-induced endothelial injury: role of Mff-based mitochondrial fission and F-actin homeostasis-mediated cellular migration. Int J Mol Med 44: 89-102.

20. Yuan T, Zhang L, Yao S, Deng SY, Liu JQ (2020) miR-195 promotes LPS-mediated intestinal epithelial cell apoptosis via targeting SIRT1/eIF2a. Int $J$ Mol Med 45: 510-518.

21. Zimmet PZ, Magliano DJ, Herman WH, Shaw JE (2014) Diabetes: a 21 st century challenge. Lancet Diabetes Endocrinol 2: 56-64. 
22. Merovci A, Solis-Herrera C, Daniele G, Eldor R, Fiorentino TV, et al. (2014) Dapagliflozin improves muscle insulin sensitivity but enhances endogenous glucose production. $J$ Clin Invest 124: 509-514.

23. Zhang J, Chen M, Chen J, Lin S, Cai D, et al. (2017) Long non-coding RNA MIAT acts as a biomarker in diabetic retinopathy by absorbing miR-29b and regulating cell apoptosis. Biosci Rep 37: BSR20170036.

24. Prado MSG, de Jesus ML, de Goes TC, Mendonça LSO, Kaneto CM (2020) Downregulation of circulating miR-320a and target gene prediction in patients with diabetic retinopathy. BMC Res Notes 13: 155.

25. Karbasforooshan H, Karimi G (2018) The role of SIRT1 in diabetic retinopathy. Biomed Pharmacother 97: 190194.
26. Zhu H, Yang Y, Wang Y, Li J, Schiller PW, et al. (2011) MicroRNA-195 promotes palmitate-induced apoptosis in cardiomyocytes by down-regulating Sirt1. Cardiovasc Res 92: 75-84.

27. Fan Y, Liu K, Wang Q, Ruan Y, Zhang Y, et al. (2014) Exendin-4 protects retinal cells from early diabetes in Goto-Kakizaki rats by increasing the Bcl-2/Bax and Bcl$\mathrm{xL} / \mathrm{Bax}$ ratios and reducing reactive gliosis. Mol Vis 20: $1557-1568$.

28. Liu S, Yang H, Hu B, Zhang M (2017) Sirt1 regulates apoptosis and extracellular matrix degradation in resveratrol-treated osteoarthritis chondrocytes via the Wnt/B-catenin signaling pathways. Exp Ther Med 14: $5057-5062$. 